\title{
EFEITOS PRODUZIDOS POR CAMADAS CONDUTORAS DE COBERTURA E ROCHA ENCAIXANTE CONDUTORA EM MEDIDAS GEOFISICAS COM MÉTODOS ELETROMAGNÉTICOS
}

\author{
JOSÉ GOUVÊA LUIZ*
}

\begin{abstract}
A review of the effects of a conducting overburden and hostrock on the electromagnetic response, based on theoretical and scale models, is presented. These results demonstrate that the interpretation of em data may be erroneous, if geological environment of the target is not taken into consideration.
\end{abstract}

INTRODUÇÃO A propagação de ondas eletromagnéticas, através de um meio, é diretamente dependente do parâmetro conhecido como constante de propagação, definido por

$$
K=\sqrt{\mu \varepsilon \omega^{2}+i \mu \sigma \omega}
$$

onde $K$ é a constante de propagação; $\mu, \varepsilon$ e $\sigma$ são respectivamente permeabilidade magnética, constante dielétrica e condutividade do meio; $\omega$ representa freqüência e $i$ o complexo $\sqrt{-1}$.

Em exploração mineral com métodos eletromagnéticos, a subsuperficie pode de um modo geral, ser representada pela seção mostrada na Fig. 1.



Figura 1 - Perfil da subsuperfície para exploração mineral com métodos eletromagnéticos: $\sigma, \varepsilon, \mu$ são respectivamente condutividade, constante dielétrica e permeabilidade magnética.

A interpretação quantitativa de dados eletromagnéticos é feita através de comparação de valores medidos no campo com valores teóricos obtidos em laboratórios, provenientes da elaboração de modelos fisicos ou matemáticos que representam a subsuperficie. A grande maioria dos valores teóricos que tem sido obtida em laboratórios, no entanto, não vem de modelos semelhantes àqueles da Fig. 1. Comumente as seguintes simplificações são efetuadas:

$$
\begin{gathered}
\sigma_{1}=\sigma_{2}=\sigma_{0} \\
\varepsilon_{1}=\varepsilon_{2}=\varepsilon_{0} \\
\mu_{1}=\mu_{2}=\mu_{0}
\end{gathered}
$$

Com isso as condutividades da camada de cobertura e da rocha encaixante são desprezadas, e a subsuperficie passa a ser aproximada por um corpo de minério envolvido pelo ar. Esta aproximação é válida em grande número de áreas e tem produzido resultados excelentes, principalmente em regiões de solo congelado como ocorre no Canadá. Por outro lado, a aplicação do modelo em outras regiões do globo, como por exemplo no oeste da Austrália (Lajoie e West, 1976), e em diversas áreas do Brasil (Palacky e Kadekaru, 1979), tem demonstrado que as condutividades do solo de cobertura e/ou da rocha encaixante (em geral da ordem de 1 a $0,001 \mathrm{mho} / \mathrm{m}$ ) são elevadas o suficiente para produzir efeitos significantes em medidas eletromagnéticas.

Neste artigo foi feita uma coletânea de modelos físicos e numéricos desenvolvidos por diversos pesquisadores. Um dos principais objetivos do artigo, é mostrar as discrepâncias que podem ocorrer na interpretação de dados eletromagnéticos, quando a condutividade da camada de cobertura e/ou da rocha encaixante é desprezada, bem como chamar a atenção dos profissionais envolvidos em interpretação, a fim de que a aplicação de ábacos seja feita com mais critério.

Camada condutora de cobertura A Fig. 2 mostra o modelo físico utilizado por Lowrie e West (1965), para estudar os efeitos de camadas de cobertura com significante condutividade.

No modelo, a camada de cobertura é representada por uma placa horizontal fina de folha de alumínio, enquanto que uma placa vertical fina de alumínio ou aço representa o condutor. As medidas foram efetuadas por um sistema de duas bobinas de eixo vertical, que registra as componentes em fase $e$

* Universidade Federal do Pará - Departamento de Geologia - Núcleo de Ciências Geofísicas e Geológicas - Caixa Postal 1611-66000 Belém-Pará - Brasil. 


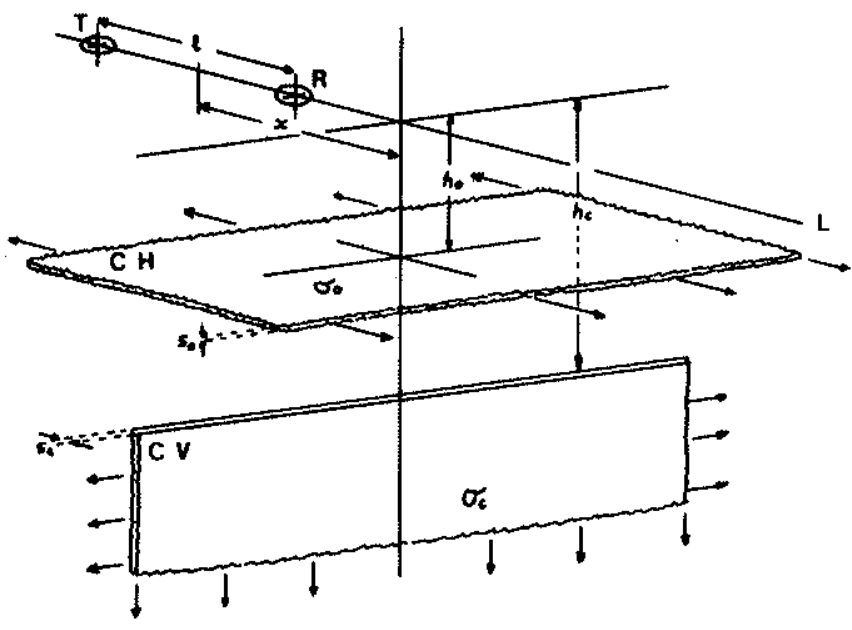

Figura 2-Geometria do modelo reduzido utilizado por Lowrie e West (1965): $T=$ transmissor; $R=$ receptor; $L=$ $=$ linha de medidas; $C H=$ condutor horizontal $C V=$ condutor vertical; $1=$ distância entre as bobinas do sistema de medidas; $H_{0}=$ profundidade do condutor horizontal; $H_{\mathrm{c}}=$ $=$ profundidade do topo do condutor vertical; $\sigma_{0}=$ condutividade do condutor horizontal; $\sigma_{\mathrm{c}}=$ condutividade do condutor vertical; $s_{0}=$ espessura do condutor horizontal; $s_{\mathrm{c}}=$ espessura do condutor vertical. Adaptado de Lowrie e West (1965).

quadratura do campo secundário expresso como percentagem do campo primário. Os resultados são apresentados nas Figs. 3 e 4, na forma de diagrama, onde as amplitudes pico a pico das componentes em fase e quadratura estão representadas.

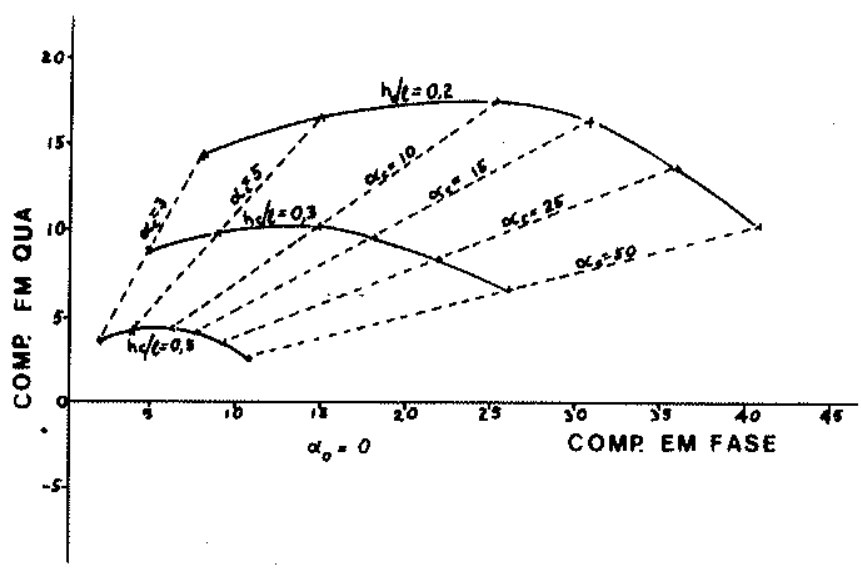

Figura 3 - Resposta de um condutor vertical na ausência de camada condutora de cobertura $\left(\alpha_{0}=0\right): \alpha_{c}=\sigma_{c} \mu_{0} \omega s_{c} 1$; $\alpha_{0}=\sigma_{0} \mu_{0} \omega s_{0} 1\left(\mu_{0}=\right.$ permeabilidade magnética do ar; $\omega=$ $=$ frequêencia). Adaptado de Lowrie e West (1965).

A Fig. 3 mostra a resposta de um condutor vertical quando não há camada de cobertura $\left(\alpha_{0}=0\right)$ para diversos valores de profundidade $\left(h_{\mathrm{c}} / 1\right.$ variável). $\mathrm{Na}$ Fig. 4 é apresentada a resposta do mesmo condutor sotoposto a uma cobertura condutora $\left(\alpha_{0}=0,25\right)$. $\mathrm{O}$ ponto $A$ na Fig: 3 tem coordenadas 10 e 15 , repre-

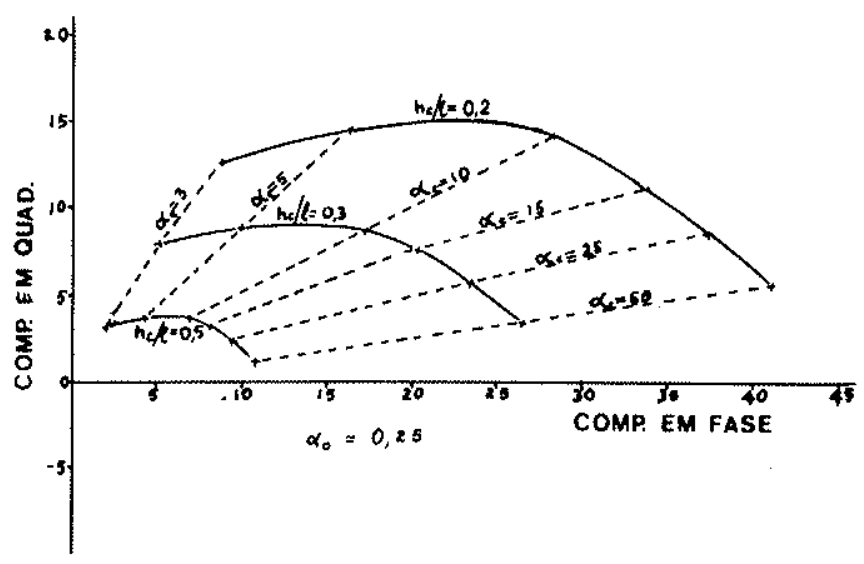

Figura 4-Resposta de um condutor vertical na presença de camada condutora de cobertura $\left(\alpha_{0}=0,25\right)$. Adaptado de Lowrie e West (1965).

sentando portanto um perfil eletromagnético anômalo em que a amplitude pico a pico da componente em fase é 10 e da componente em quadratura é 15 . O referido ponto repousa sobre a curva $h_{\mathrm{c}} / 1=0,2$, o que leva a uma interpretação de profundidade para o condutor de um valor igual a 0,2 multiplicado pela distância entre as bobinas de medida. Esse mesmo ponto está representado na Fig. 4. Neste caso, no entanto, o ponto não mais repousa sobre a curva $h_{\mathrm{c}} / 1=0,2$ e deverá, portanto, levar a uma interpretação de profundidade diferente da anterior.

Comparando as Figs. 3 e 4, pode-se ainda concluir que as amplitudes das anomalias são reduzidas e as fases sofrem uma rotação horária com o aumento da condutividade de camada de cobertura.

Lajoie e West (1976) alcançaram resultados idênticos aos de Lowrie e West, modelando numericamente uma cobertura condutora e simulando o sistema eletromagnético TURAM.

Rocha encaixante condutora Modelos reduzidos físicos foram utilizados por Verma e Gaur (1975) para estudar o efeito de encaixante condutora em medidas eletromagnétiças sobre condutores inclinados. As Figs. 5 e 6 mostram alguns dos resultados obtidos no modelamento.

$\mathrm{Na}$ Fig. 5 um condutor vertical, imerso em um meio não condutor, apresenta anomalia simétrica, enquanto que o condutores com mergulho diferente de $90^{\circ}$ imersos no mesmo meio não condutores respondem assimetricamente. Por outro lado, em um meio condutor, a resposta é simétrica para qualquer valor de mergulho (Fig. 6); deve ser enfatizado que condutores horizontais tendem a aparecer verticais com o aumento da condutividade da rocha encaixante.

Outros resultados obtidos por Verma e Gaur (1975), mostram que os efeitos de uma encaixante condutora são mais pronunciados em sistemas de medidas com bobinas complanares horizontais do que em sis- 

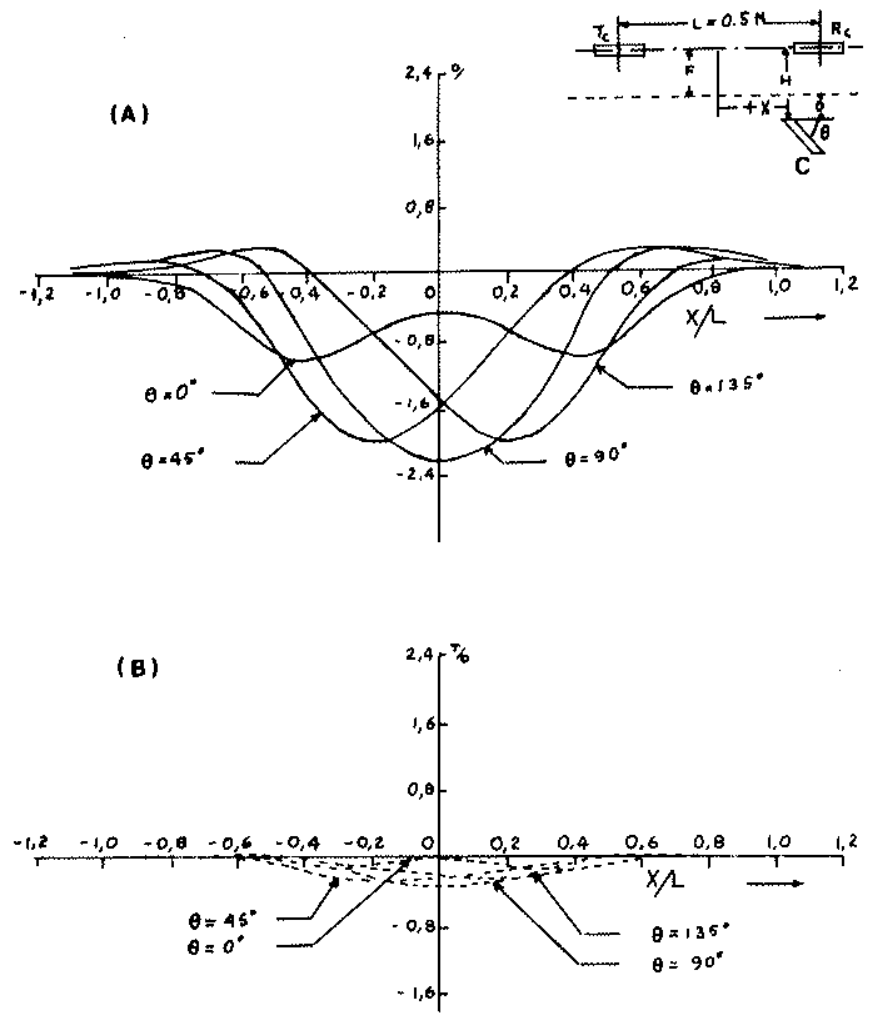

Figura 5-Resposta para diversos valores de inclinação do condutor $C$ imerso no ar: A) componente em fase; B) componente em quadratura. $T_{\mathrm{d}}=$ bobina transmissora; $R_{\mathrm{c}}=$ = bobina receptora; $F=0,17 \mathrm{~m} ; H=0,22 \mathrm{~m} ; D=0,05 \mathrm{~m}$; condutividade da placa representando o condutor $=26,3 \times$ $\times 10^{3} \mathrm{mho} / \mathrm{m}$; condutividade do meio envolvente $(\mathrm{ar}) \simeq 0$; frequiência $=100 \mathrm{kHz}$; fator escala do modelo reduzido $=$ $=500$. Adaptado de Verma e Gaur (1975).

temas de bobinas verticais, e que as anomalias mais proeminentes são registradas nos sistemas de bobinas horizontais.

Lajoie e West (1976), modelando numericamente medidas efetuadas pelo sistema TURAM sobre condutores verticais, encontraram que a fase da anomalia é rotacionada no sentido horário e sua amplitude é aumentada como resultado do aumento de condutividade da rocha encaixante.

\section{Camada condutora de cobertura e encaixan-} te çondutora O efeito conjunto de camada condutora de cobertura e encaixante condutora foi estudado por Lajoie e West (1976), utilizando modelamento numérico. O sistema de medidas TURAM e condutores verticais foram considerados (Fig. 7).

Os resultados de Lajoie e West são mostrados através do diagrama da Fig. 8, onde estão representados os valores pico a pico das componentes em fase e quadratura das anomalias. O diagrama evidencia um aumento na amplitude das anomalias e uma rotação horária nas fases provenientes do aumento da condutividade da rocha encaixante.



Figura 6-Resposta da componente em fase para diversos valores de inclinação do condutor $C$ imerso em um meio condutor; $T_{\mathrm{c}}=$ bobina transmissora; $R_{\mathrm{c}}=$ bobina receptora ; $F=0,17 \mathrm{~m} ; H=0,22 \mathrm{~m} ; D=0,05 \mathrm{~m}$; condutividade da placa representando o condutor $=26,3 \times 10^{3} \mathrm{mho} / \mathrm{m}$; condutividade de solução representando o meio envolvente $=$ $=18 \mathrm{mho} / \mathrm{m} ; S=$ nivel da solução; frequiência $=100 \mathrm{kHz}$; fator escala do modelo reduzido $=500$. Adaptado de Verma e Gaur (1975).

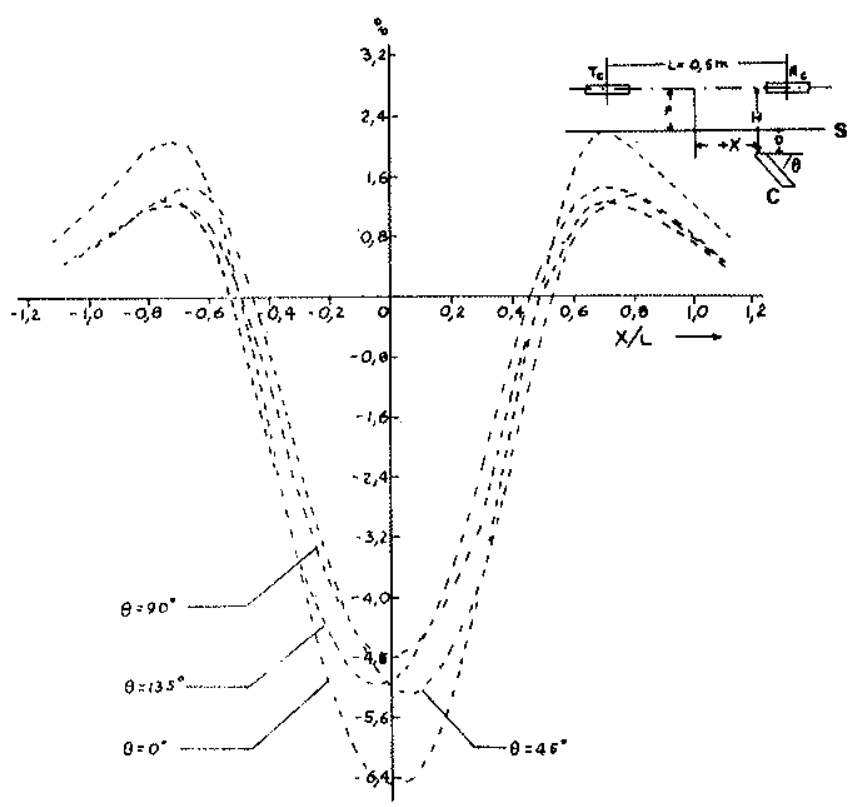

Figura 6B - Resposta da componente em quadratura para diversos valores de inclinação do condutor $C$ imerso em um meio condutor. As condutividades, frequiência e parâmetros geométricos utilizados são os mesmos descritos na Fig. 6. Adaptado de Verma e Gaur (1975). 




Figura 7-Geometria do modelo estudado por Lajoie e West (1976): $T=$ transmissor (freqüência $=500 \mathrm{~Hz}$ ); $\sigma_{0}=$ condutividade do $\operatorname{ar}=0 ; \sigma_{\mathrm{L}}=$ condutividade da camada de cobertura $; \sigma_{\mathrm{H}}=$ condutividade da rocha encaixante; $\sigma_{\mathrm{R}}=$ condutividade do corpo condutor. Adaptado de Lajoie e West (1976).

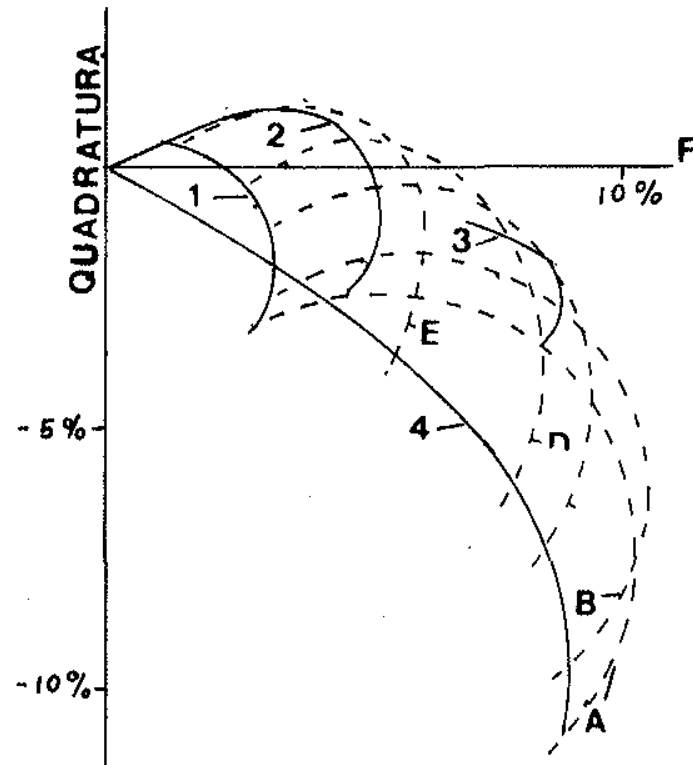

Figura 8 - Resposta obtida com o modelo da Fig. 7. A condutividade do corpo e da encaixante são variáveis; a condutividade da camada de cobertura é $2,0 \times 10^{-2} \mathrm{mho} / \mathrm{m}$. As linhas cheias representam valores em que $\sigma_{\mathrm{H}}$ é constante, enquanto que as linhas tracejadas representam valores de $\sigma_{\mathrm{R}}$ constante. Adaptado de Lajoie e West (1976).

\section{CONCLUSOES Os resultados apresentados de-} monstram que:

a) Quando um condutor está sotoposto a uma condutora e/ou envolvido por encaixante condutora, a amplitude e a fase de sua anomalia é em geral bem diferente da anomalia produzida quando imerso em um meio não condutor.

b) As componentes em fase e a quadratura da anomalia, tornam-se significantemente pronunciadas com o aumento da condutividade da rocha encaixante.

c) A interpretação utilizando o ar como meio envolvente do condutor, quando uma camada condutora e/ou encaixante condutora está presente, pode produzir um efeito tal que um mau condutor é interpretado como bom condutor. Esse efeito vem da rotação da fase da anomalia em direção da componente em fase (rotação no sentido horário). Além disso, o condutor interpretado parecerá estar mais profundo do que ele realmente está (Lowrie e West, 1965).

Finalmente, é sugerido um cuidado especial na aplicação de ábacos teóricos na interpretação de dados geofisicos de um modo geral (não só de dados eletromagnéticos), devendo ser escolhidos aqueles desenvolvidos através de modelos reduzidos que se assemelham ao modelo geológico da área em exploração. As Figs. 9 e 10 demonstram as deficiências dos esquemas de interpretação baseados nos modelos simples (no ar). Tendo em vista, no entanto, que algumas vezes é dificil conseguir ábacos que produzam o ambiente geológico das áreas do Brasil, pois os ábacos são em geral preparados em outros países, sugere-se às companhias envolvidas em exploração mineral que apliquem em pesquisa junto a universidades brasileiras, com $o$ intuito de desenvolver pacotes de interpretação apropriados às áreas geológicas brasileiras. Existem já no Brasil entidades perfeitamente equipadas para desenvolver tais pacotes; como, por exemplo, o Núcleo de Ciências Geofisicas e Geológicas da Universidade Federal do Pará. 


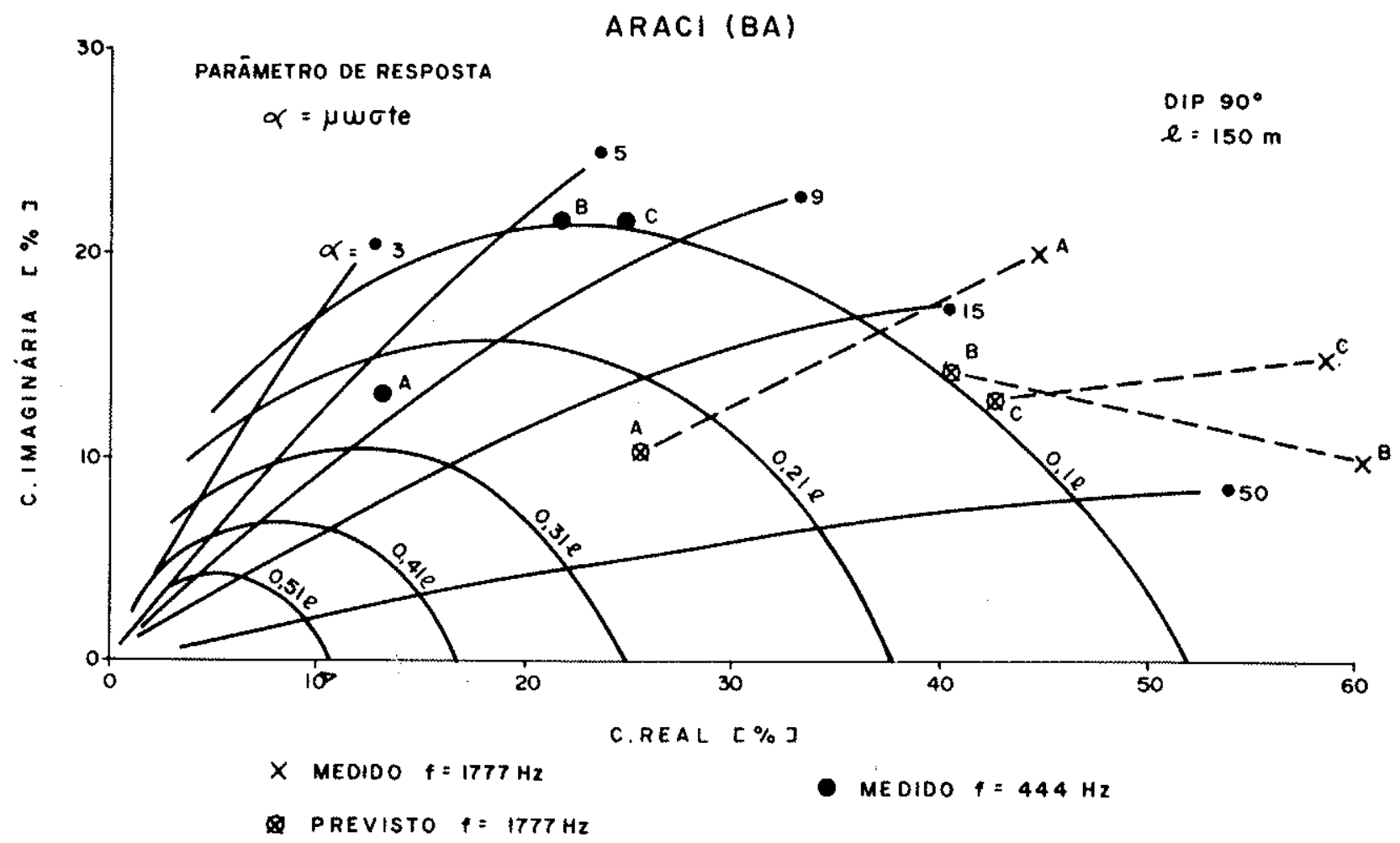

Figura 9 - Diagrama de Argand e estimativas de profundidade e condutância para anomalias $A, B$ è $C$. Adaptado de Palacky e Kadekaru (1978).

\section{FREQUENCY MIGRATION \\ $222 \mathrm{~Hz}$ to $3555 \mathrm{~Hz}$}

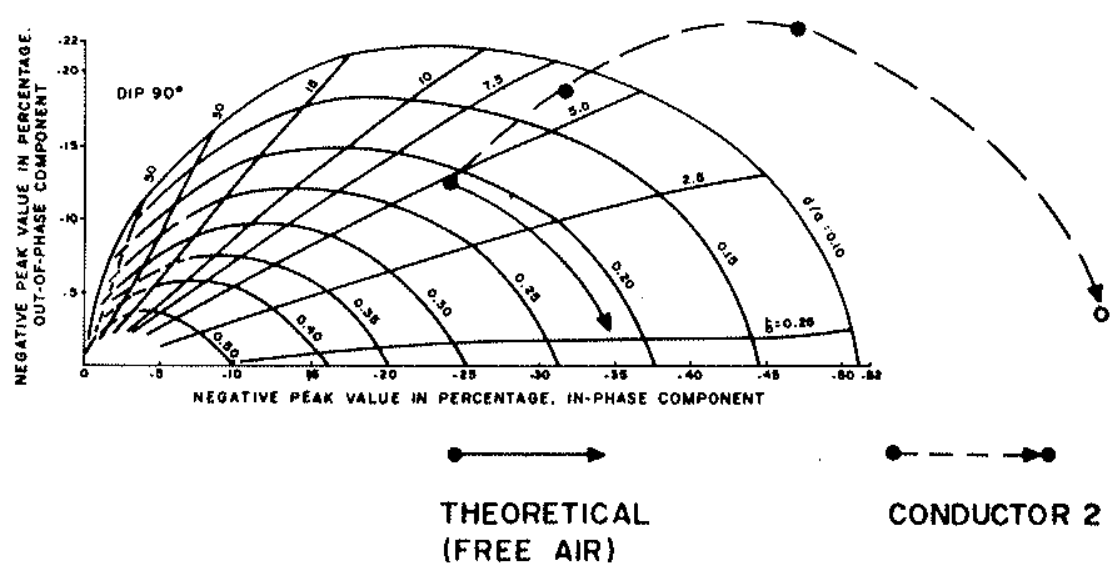

Figura 10 - Migração teórica da anomalia com a mudança de freqüência é comparada com aquela observada para o condutor. Adaptado de Lajoie e West (1977).

\section{BIBLIOGRAFIA}

LAJOIE. J.J. e WEST. G.F. - 1976 - The EM response of a conductive inhomogeneity in a layered earth. Geophysics; 41: 1133-1156.

LAJOIE, J.J. and WEST, G.F., - 1977 - Two selected ficld exampies of EM anotnalies in a conductive environment, short note: Geophysics, 42: $655-660$.

LOWRIE, W. e WEST, G.F. - 1965 - The effect of a conducting overburden on EM prospecting measurements. Geophysics. 30: 624-632.

PALACKY, G.J. and KADEKARU, K., - 1978 - Eleito do intemperis- mo tropical nos levantamentos eletromagnéticos: Anais do XXX Congresso Brasileiro de Geologia, Recife, Volume V.

PALACKY. G.J. e KADEKARU. K., - 1979-Elfect of tropical weathering on electrical and electromagnetic measurements. Geophysics; 44 69-88.

VERMA, O.P. e GAUR, V.K., -- 1975 - Transformation of EM anomalies brought about by a conducting host rock. Geophysics, 40: 473-489. 